\title{
Anode Buffer Layer containing Au Nanoparticles for High Stability Organic Solar Cells
}

\author{
Ryo Morioka ${ }^{1}$, Kei Yasui ${ }^{3}$, Masaaki Ozawa ${ }^{3}$, Keisuke Odoi ${ }^{3}$, \\ Hisashi Ichikawa ${ }^{2}$, and Katsuhiko Fujita ${ }^{1,2}$ *
}

${ }^{1}$ Department of Applied Science for Electronics and Materials, Graduate School of Engineering Sciences, Kyushu University, 6-1 Kasuga Koen, Kasuga, Fukuoka 816-8580, Japan

${ }^{2}$ Institute for Materials Chemistry and Engineering, Kyushu University, 6-1 Kasuga Koen, Kasuga, Fukuoka 816-8580, Japan

${ }^{3}$ Nissan Chemical Industries, Ltd, 3-7-1 Kinshityou, Kanda, Chiyoda-ku, Tokyo 101-0054, Japan

\begin{abstract}
For a promising approach to improve the energy conversion performance of organic thin layer solar cells, a buffer layer between a hole collecting electrode (anode) and organic active layer containing electron accepting and donating materials mixture is investigated. A conductive polymer, PEDOT:PSS, is generally used as the buffer layer to realize the best performance. However, it often causes degradation in long term stability due to its highly acidic and absorbent nature. In the present study, we investigate properties of a new class of buffer materials substituting PEDOT:PSS, a composite of gold nanoparticles and hyper-branched polystyrene.
\end{abstract}

\section{Keywords : organic solar cells, anode buffer layer, hyper-branched polymers, nanoparticle}

\section{Introduction}

Organic solar cells (OSCs) are attracting much attention as a solar cell for the next generation due to the potentially low-cost, light-weight and mechanically flexible nature. Though they have not been commercial products yet, over $6 \%$ of the power conversion efficiency (PCE) has been reported [1].

In general, poly(3,4-ethylenedioxythiophene): poly(4-styrenesulfonate) (PEDOT:PSS) is used as an anode buffer layer on the transparent electrode, indium tin oxide (ITO), in polymer based OSCs showing high PCE. However, it has been reported that PEDOT:PSS often causes degradation of the cells due to the highly acidic and absorbent nature [2] [4]. To put them to practical use, it should be necessary to develop a new class of the buffer layer materials which is applicable to various low-cost production processes and does not cause degradation in long term stability.

In the present study, we investigate the buffer layer ability of a composite of gold nanoparticles (Au-NPs) and hyper-branched polystyrene (HPS) which can be used as a hole injection material in organic light emitting diodes [5]. The composite can be processed into the buffer layer by a conventional spin-coat of the solution and contains no acidic ingredient. The typical bulkhetero-junction type OSCs composed of poly(3-hexylthiophene) (P3HT)

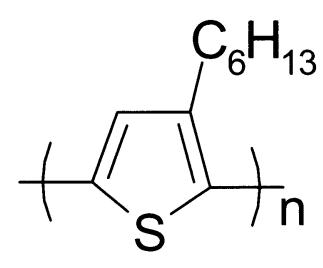

P3HT

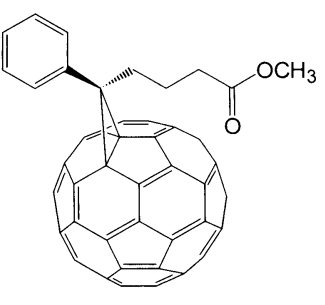

PCBM
Figure 1. Molecular structures of P3HT and PCBM.

and [6,6]-phenyl-C61-butyric acid methyl ester (PCBM), molecular structure in Fig. 1, with the composite buffer layer or PEDOT:PSS were fabricated and investigated in the device durability.

\footnotetext{
Received April 10, 2010

Accepted May 14, 2010
} 


\section{Experimental}

The device structure fabricated is shown in Fig.2. The device without buffer layer (A), with PEDOT:PSS buffer layer (B) and with HPS composite buffer layer (C) were fabricated.

The ITO substrate was cleaned by a UV-ozone treatment (NL-UV253, Nippon Laser Denshi). PEDOT:PSS (AI4083: H. C. Starck) was placed onto a pre-cleaned ITO substrate through PVDF syringe filter with $0.45 \mu \mathrm{m}$ pore and spun at 2000 rpm for $30 \mathrm{sec}$, followed by thermal treatment at $130{ }^{\circ} \mathrm{C}$ for $20 \mathrm{~min}$.

Au-NPs were synthesized as previously reported [5]. They were 2-3 nm diameter and coated and stabilized by HPS. The composite of HPS and Au-NPs, which contains Au in $4.5 \mathrm{wt} \%$ (by ICP), was dissolved in $o$-dichlorobenzene at the concentration of $0.3 \mathrm{wt} \%$ and placed onto a pre-cleaned ITO substrate through a PTFE membrane filter with $0.50 \mu \mathrm{m}$ pore and spun at $3000 \mathrm{rpm}$ for $30 \mathrm{~s}$, followed by thermal treatment at $150{ }^{\circ} \mathrm{C}$ for $30 \mathrm{~min}$ and UV-radiation by a high pressure mercury lamp for $30 \mathrm{~min}$ to harden the polymer. The substrate was treated by UV-ozone again before the next step.

The mixture solution of the electron donating material, P3HT and the electron accepting material, PCBM, $(1: 1, \mathrm{w} / \mathrm{w})$ in $o$-dichlorobenzene at the concentration of $3.0 \mathrm{wt} \%$ was placed on the substrate with and without the buffer layer through a PTFE membrane filter with $0.50 \mu \mathrm{m}$ pore and spun at the sequence of $1 \mathrm{st}: 500 \mathrm{rpm}, 30 \mathrm{sec}, 2 \mathrm{nd}$ : $2000 \mathrm{rpm}, 1 \mathrm{sec}, 3 \mathrm{rd}: 500 \mathrm{rpm}, 300 \mathrm{sec}$, followed by thermal treatment at $120{ }^{\circ} \mathrm{C}$ for $10 \mathrm{~min}$. The $\mathrm{LiF}$ $(0.5 \mathrm{~nm}) / \mathrm{Al}(150 \mathrm{~nm})$ was deposited as cathode by a vacuum evaporator to result in the active area of 2 $\mathrm{mm} \times 2 \mathrm{~mm}$.

The device $\mathrm{A}, \mathrm{B}$ and $\mathrm{C}$ were placed in a dark ambient atmosphere for 100 hours to investigate the time-dependence of the device performance.

The separately fabricated device A, B and C were sealed with a glass plate and UV-curing resin in a dry nitrogen environment and placed in a dark for 400 hours to see the degradation of the cells in the inert atmosphere.

The photovoltaic performance was measured by the source-measure unit (Keithley 238) at the range of $-1.5 \mathrm{~V}$ to $1.5 \mathrm{~V}, 0.05 \mathrm{~V} / \mathrm{sec}$ with and without light irradiation of AM 1.5 at $100 \mathrm{~mW} / \mathrm{cm}^{2}$ by a solar simulator YSS-50A (Yamashita denso) with 2 $\mathrm{mm} \times 2 \mathrm{~mm}$ shadow mask.

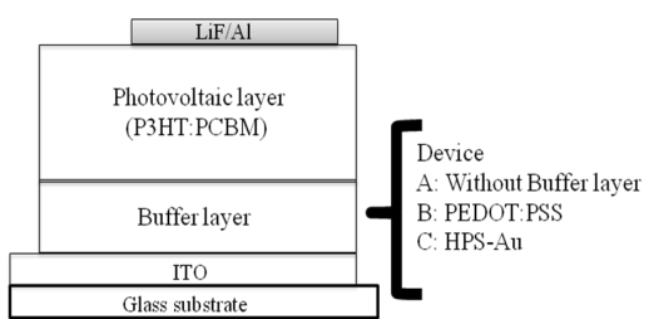

Figure 2. Schematic device structure of organic solar cell used in this study

\section{Results and discussion}

The initial $\mathrm{J}-\mathrm{V}$ characteristics and performance of the device A, B and C were summarized in Fig. 3 and Table 1. The device $C$ with the HPS composite buffer layer showed almost comparable performance to the device B with the conventional PEDOT:PSS buffer layer, while the short circuit current (Jsc) and open circuit voltage (Voc) was slightly lower and the fill factor (FF) was slightly higher, which suggests the slightly lower conductivity and higher rectification. In both cases the performance was improved drastically compared with the device A.

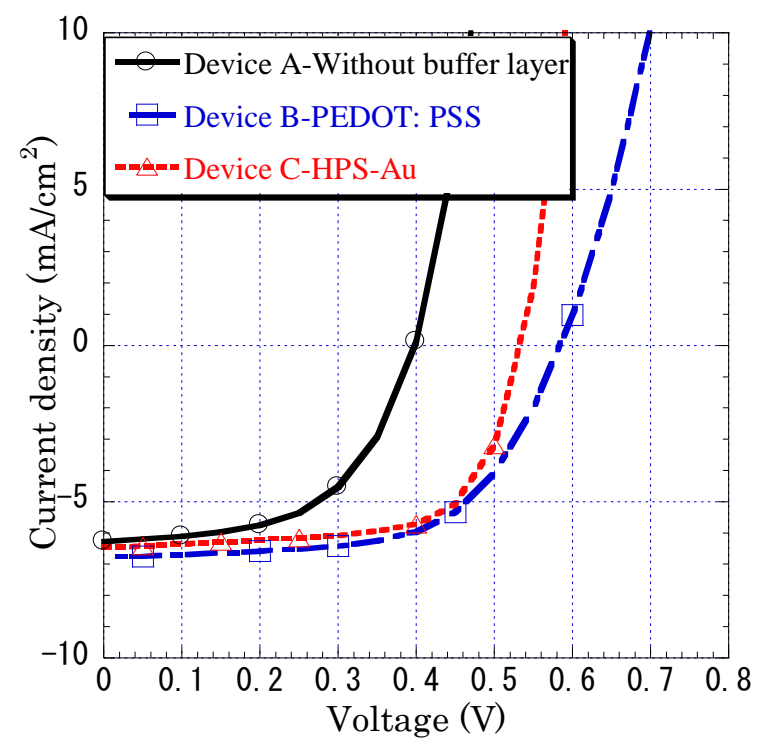

Figure 3. The J-V characteristics of the Device A (circles), the Device B (squares), and the Device C (triangles) and light (AM 1.5) conditions, respectively.

The J-V characteristics of the device A, B and C placed at a dark ambient atmosphere for 20, 50 and 100 hours (Fig.4) clearly showed that the performance got lowered as the time. Time course is also showed in Figure 5. 
Table 1. Device parameters of the three devices

\begin{tabular}{cccccc}
\hline Device & $\begin{array}{c}\text { Buffer } \\
\text { layer }\end{array}$ & $\begin{array}{c}\mathrm{J}_{\mathrm{sc}} \\
\left(\mathrm{mA} / \mathrm{cm}^{2}\right)\end{array}$ & $\mathrm{V}_{\mathrm{oc}}(\mathrm{V})$ & $\mathrm{FF}$ & PCE (\%) \\
\hline A & - & 6.26 & 0.40 & 0.55 & 1.36 \\
B & PEDOT & 6.76 & 0.58 & 0.61 & 2.39 \\
C & HPS-Au & 6.38 & 0.54 & 0.68 & 2.32 \\
\hline
\end{tabular}
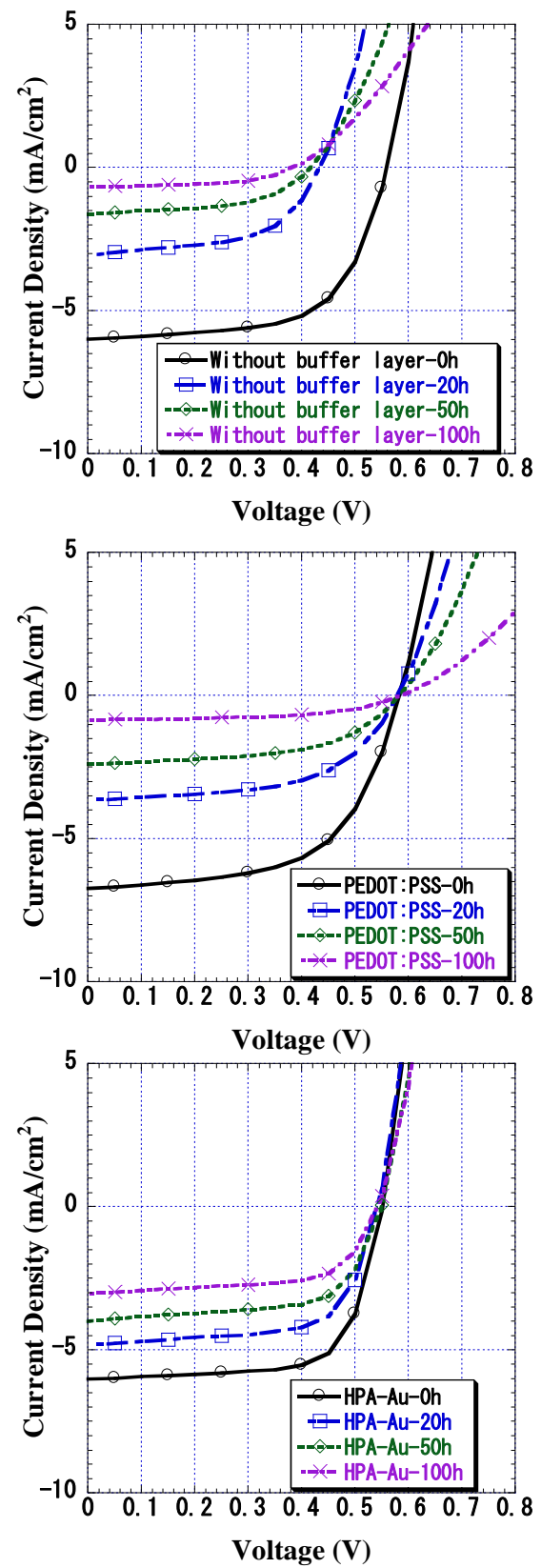

Figure 4. The J-V characteristics of device A, B and C at $0,20,50$ and 100 hours after the fabrication.

The performance of device A was degraded in Jsc, Voc and FF worst among three to $20 \%$ of the initial values after 100 hours. In the device B and $\mathrm{C}$, the Voc was almost constant but the Jsc was getting lower. The device $\mathrm{C}$ showed significantly more moderate degradation. it might proceed much slower than in the device B where the acidic PSS corrosively interact mainly with the upper electrode after adsorbing moisture.

The large degradation of Voc in the device A might be caused by the accumulated carrier at the anode interface bringing about photochemical reaction of the active materials.
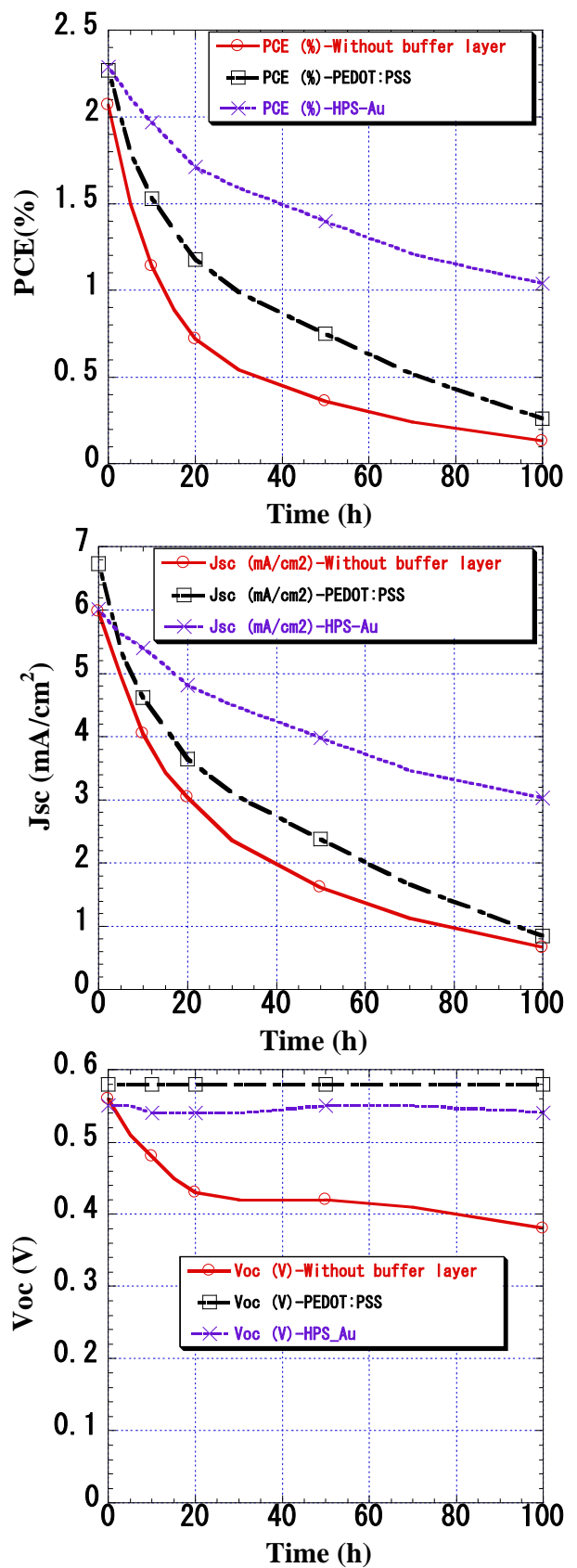

Figure 5. Time course of PCE, Jsc and Voc in device A, $\mathrm{B}$ and $\mathrm{C}$.

The device sealed with glass and resin in dry nitrogen showed much slower degradation than the devices placed in ambient atmosphere. In the 

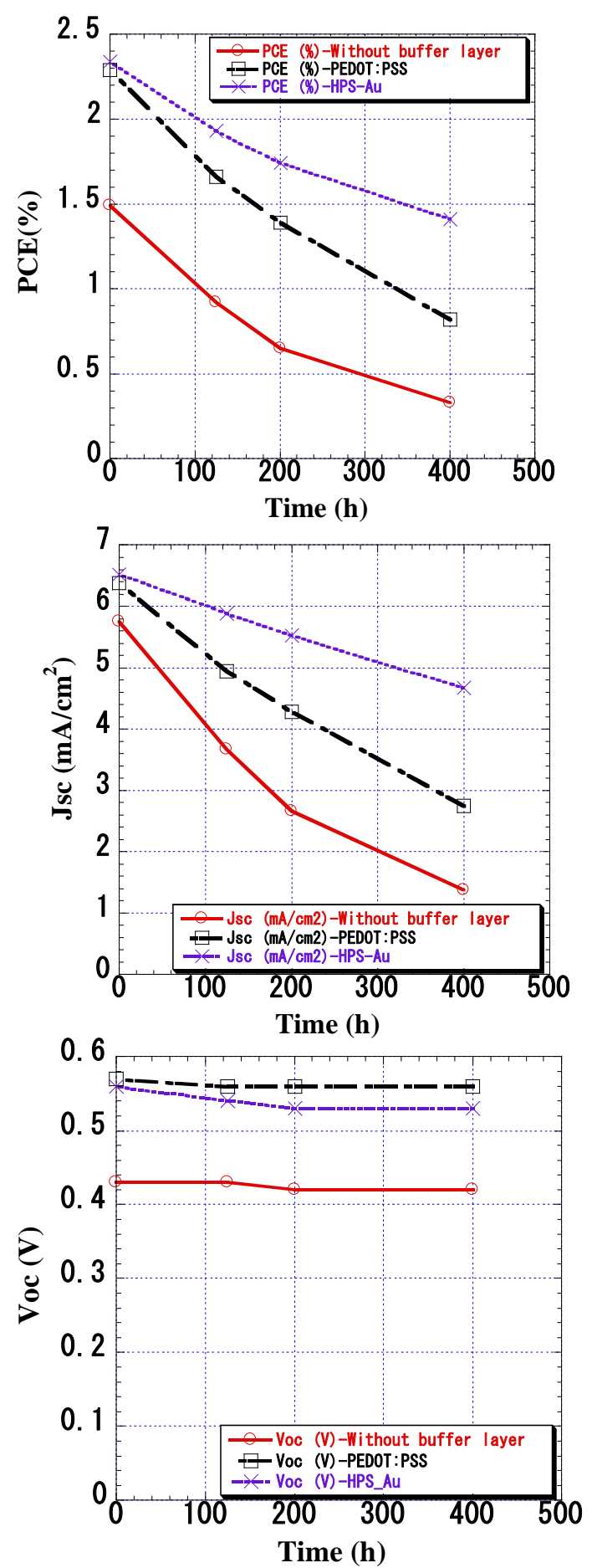

Figure 6 Time course of PCE, Jsc and Voc in device A, $\mathrm{B}$ and $\mathrm{C}$ with sealed with nitrogen. inert environment as well, the deice $\mathrm{C}$ showed slowest degradation (Fig.6). In the deice B and C, the degradation rate was suppressed drastically, however, device A without buffer layer showed much less improvement. The effect of the accumulated carrier at the anode interface to the degradation might be relatively independent of the environment.

In summary, the HPS-Au nano composite layer can be used as the anode buffer layer and showed almost comparable initial performance and much more durability than PEDOT:PSS probably due to no acidic ingredient in the buffer layer.

\section{References}

1. Hsiang-Yu Chen.; Jianhui Hou.; Shaoqing Zhang.;Yongye Liang.; Guanwen Yang.; Yang.; Luping Yu.; Yue Wu. ; Gang Li. Nature Photonics., 25 (2009) 649.

2. Kawano, K.; Pacios, R.; Poplavskyy, D.; Nelson, J.; Bradley, D.; Durrant, J. R. Solar Energy Materials \& Solar Cells , 90 (2006) 3520-3530.

3. Danier Van Der Gon, A. W.; Birgerson, J.; Fahlman, M.; Salaneck, W. R. Org. Electr., 3 (2002) 111-118.

4. Greczynski, G.; Kugler, T.; Keil, M.; Osikowicz, W.; Fahlman, M.; Salaneck, W. R. J. Elec. Spectr. \& Rel. Phenom. (2001) 121 1-17.

5. Ichikawa H.; Yasui K.; Ozawa M.; Fujita K. Synth. Met., 159 (2009) 973-976 